\title{
Layer-based Dome Contents Creation Using Scenario Description Language
}

\author{
Tetsuro Ogi, Daisuke Furuyama \\ Graduate School of System Design and Management \\ Keio University \\ 4-1-1 Hiyoshi, Kohoku-ku, Yokohama, Japan \\ ogi@sdm.keio.ac.jp,j6304068@z8.keio.jp
}

\author{
Tetsuro Fujise \\ Mitsubishi Research Institute, Inc. \\ 2-3-6 Otemachi, Chiyoda-ku, Tokyo, Japan \\ fujise@mri.co.jp
}

\begin{abstract}
In recent years, dome display has become popular, and it is desired to establish a method to create dome image contents easily. In this study, psychophysical experiment was conducted to measure the three-dimensional sensation that was felt in the dome environment quantitatively. Based on the result of this experiment, the layered image representation method was developed. In this method, dome image is generated by placing the two-dimensional layered images in the three-dimensional space. The user can feel the threedimensional sensation from the image by moving the layers or view position effectively. In addition, the scenario description language and the renderer were developed so that the usual creator can produce the dome image contents easily without the programming. This method was applied to produce some dome image contents and the effectiveness of this method was evaluated.
\end{abstract}

Keywords-dome display; planetarium; motion parallax; depth sensation; scenario description language

\section{INTRODUCTION}

In the past years, the number of customers has been decreased in many planetariums due to the diversification of the amusement facility. However, the recent digitalization of the planetarium facilities has diversified the usage. In the digital planetarium, it has become possible to project not only the constellation but also three-dimensional image, because the computer generated image is projected onto the dome screen using the high definition projectors [1][2]. And then, many people have become interested in planetarium contents.

On the other hand, dome display such as I-MAX Dome [3], CyberDome [4] or Earth Room exhibited in Aichi Expo [5] has become popular as an immersive display environment in which a large number of people can experience the omni-directional image. These dome displays can be used to project the three-dimensional stereo image. However, in the current production method of the dome image contents, it is a problem that a large amount of time and costs are required to create the contents, because the know-how of creating effective image often depends on the experience rule in the specific creator.

Therefore, the purpose of this study is to establish a methodology of creating a three-dimensional image easily for the dome display environment that includes the planetarium facility and to generate a contents industry for the dome display. In this case, the word "easily" means that the creators can create the contents without programming and specific experience rule as well as that the audiences can see the three-dimensional image without using the special stereo glasses.

In this paper, the principle of generating the threedimensional image space and the basic experiment concerning the depth perception for the dome image are described. After that, the contents creation method of the layered image representation that was proposed in this study and the scenario description language that was developed to create the contents without the programming are discussed.

\section{DEPTH SENSATION IN DOME ENVIRONMENT}

It is known that the user in the dome space can feel the depth sensation from the frameless image with wide field of view that is projected onto the curved screen [6]. For example, in the condition where the shape of the dome screen is recognized by the audience, the projected image is felt as a distorted image on the curved screen. However, when the geometrically corrected image is projected in the dark condition where the audience cannot see the screen shape, he can perceive the correct image without the distortion.

It seems that this is because the top down thinking that the image should be projected onto the plane screen would become predominant when the audience could not recognize the screen shape. In addition, when the images of the displayed objects were moved on the virtual plane, the sense of seeing the three-dimensional image would become strong based on the effect of motion parallax, and the audience can perceive that the image is floating in the air. Figure 1 shows the above-mentioned principle of perceiving the threedimensional sensation from the displayed image in the dome environment.

This three-dimensional sensation can be felt without using the binocular parallax, and it is caused by the fact that several factors that have influence on the human perception of the three-dimensional shape are not equivalent. Namely, we can consider that the effect of motion parallax and the perspective view of the experience factor worked more dominantly than other factors under the condition of wide field of view. Though this effect that enable the user to perceive the depth sensation from the projected image in the dome environment has been known in experience, the quantitative characteristic has not been analyzed and it has not been used as image production technology. 


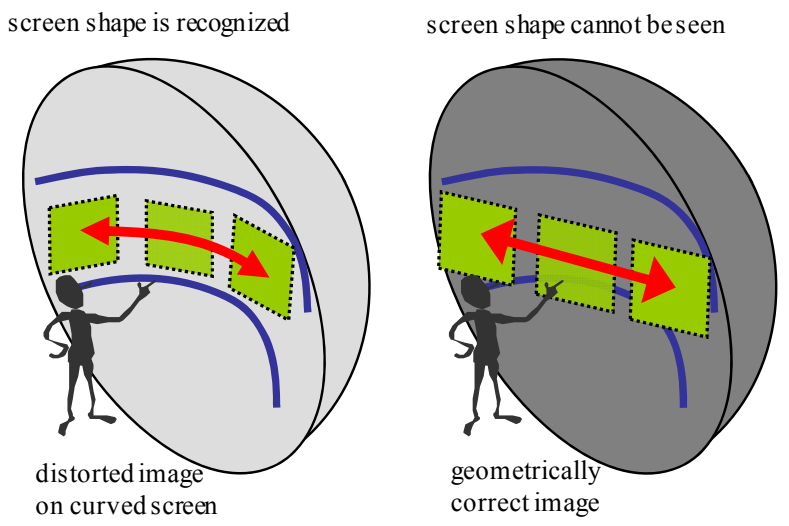

Figure 1. Principle of perceiving depth sensation in dome environmen.

This study aims at systematizing the knowledge of the three-dimensional sensation that is perceived in the dome display environment based on the psychological experiment and utilizing it as contents creation technology. Particularly, the depth perception that was felt when the image moved along the virtual surface was quantitatively evaluated in the psychophysical experiment.

\section{EXPERIMENT ON DEPTH PERCEPTION}

In this study, the following psychophysical experiment was conducted to quantitatively evaluate the depth sensation that was felt from the moving image in the dome environment. As for the experimental environment, the inclined type planetarium with $18 \mathrm{~m}$ in diameter Hokutopia was used. In this system, one LCD projector NEC NP2000J with high luminance of 4000 lumens equipped with the fisheye lens RAYNOX DCR-CF185PRO was used, so that the image with 130 degree horizontal angle and 100 degree vertical angle was projected onto the dome screen. Figure 2 shows the experimental environment.

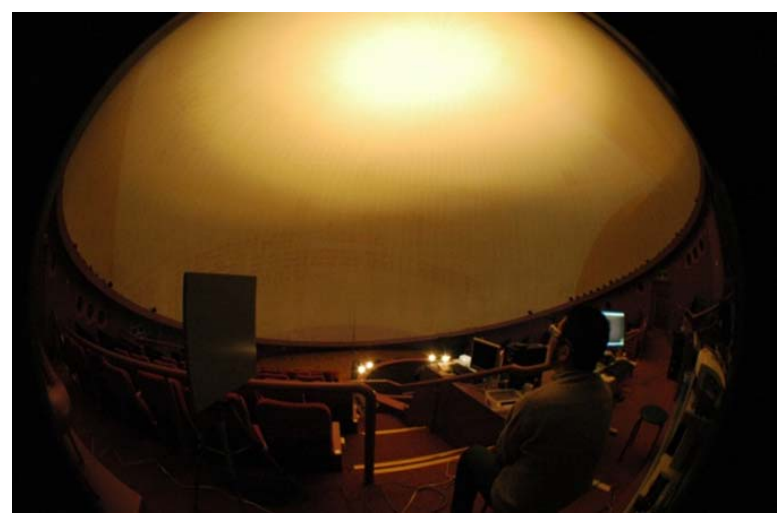

Figure 2. Experimental environment using a planetarium.

In the experiment, the subjects were asked to sit on the chair at the distance of $11 \mathrm{~m}$ from the front of the screen and to answer the distance that was felt from the projected image using the magnitude estimation method. As a standard stimulus for the value of 100 , the distance to the stick that is standing at the front screen was used [7].

As a comparison stimulus, 36 patterns of images were projected six times respectively, in combinations of four factors such as kinds (square and human), sizes (large, medium and small), backgrounds (floor, ceiling and nothing), and view positions (fixed and moving). Figure 3 shows the examples of the displayed images. The distortion of the projected images caused by the fisheye lens and the curved screen were corrected by the software. Each image moved right and left on the virtual plane placed at $11 \mathrm{~m}$ ahead from the subject taking 14 seconds.

In this experiment, the kinds of the displayed images were selected to compare the images of the object that has a certain size (human) and the object that has not a certain size (square). The sizes of the images represented on the virtual plane were selected from large $(9 \mathrm{mx} 9 \mathrm{~m})$, medium $(6 \mathrm{mx} 6 \mathrm{~m})$ and small $(3 \mathrm{~m} \times 3 \mathrm{~m})$. In addition, the backgrounds of floor, ceiling and nothing were changed to examine the influence of the reference object on the depth perception of the target image. The view positions of fixed and moving mean that the moving objects is seen from the fixed view position or seen from the moving view position along with the moving objects. This condition of the moving view position was investigated to examine the influence of the camera work in the dome environment.

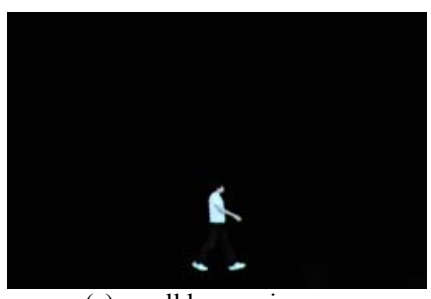

(a) small human image

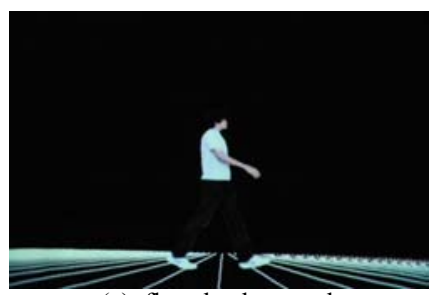

(c) floor background

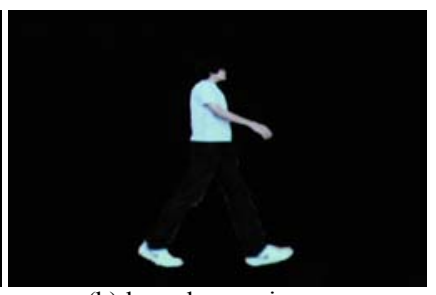

(b) large human image

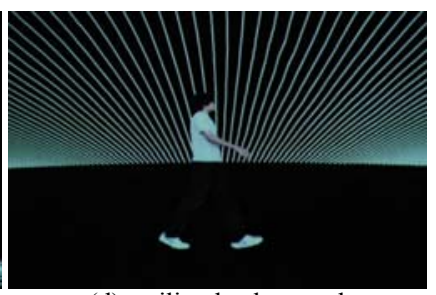

(d) ceiling background
Figure 3. Examples of displayed images in the experiment.

The results of the experiment were obtained for eleven subjects, and the analysis of variance was applied to test the four factors. In Figure 4, the mean value and the standard error of the perceived distance value for each size of the displayed image are shown. As for the size, significant difference at the one percent level was found between large and medium, and between medium and small. From the result, we can see that the subjects felt the difference of the depth sensation according to the difference of the size, when the geometrically corrected image was moved on the virtual plane even if it was seen without the effect of binocular parallax. 
In Figure 5 to Figure 7, the mean value and the standard error for each factor in each size are shown to investigate the influence of these factors. As for the kinds, though the significant difference was found between square image and human image when the image size was small, there were no differences between them when the image size was medium or large. It seems that this is because the subjects had impression of seeing the image of wall, when the square image was displayed. As for the background, when the size was large or medium, the image with the ceiling background was perceived at nearer distance, and image with the floor background was felt at farther. It can be considered that this result was caused by the distance of the screen position on which the background image was projected, because the image of the ceiling was projected above the head of the subjects and the image of the floor was projected onto the screen area that is the farthest from the subjects. The result on the view position indicates that there was no significant difference between fixed view position and moving view position. Namely, we can see that the movement of the background image due to the camera work generates almost same effect as the movement of the object.

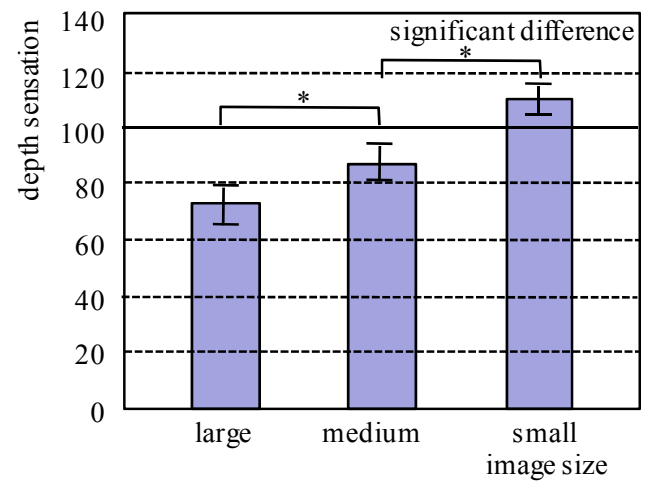

Figure 4. Perceived depth sensation for image size .

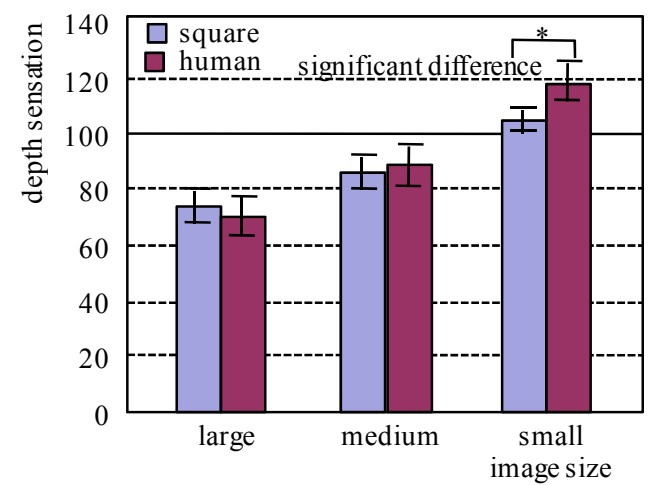

Figure 5. Perceived depth sensation for image size and kind .

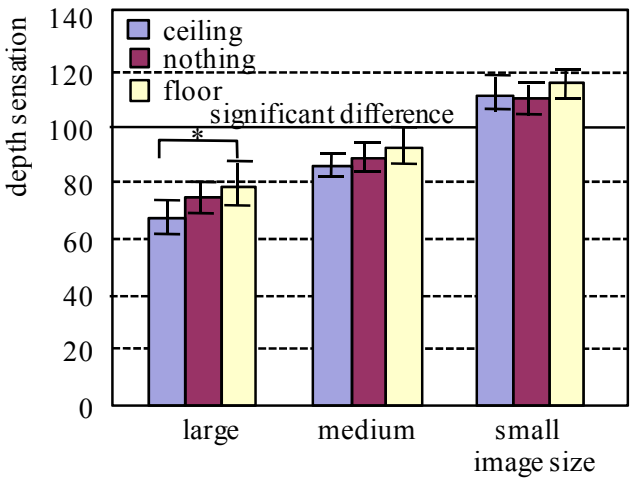

Figure 6. Perceived depth sensation for image size and background .

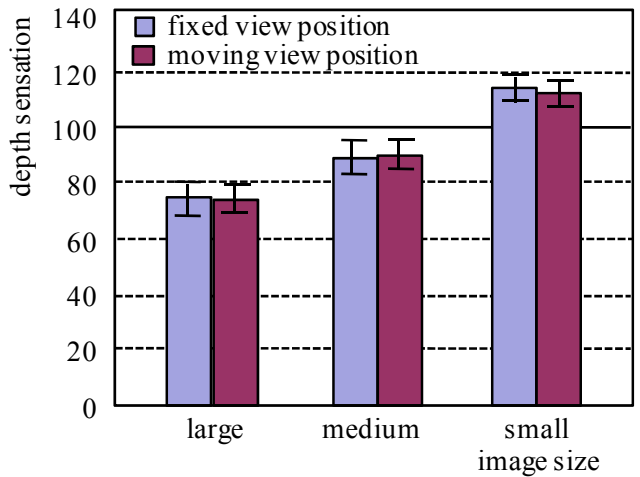

Figure 7. Perceived depth sensation for image size and view position.

\section{LAYER-BASED DOME IMAGE}

From the psychophysical experiment, we can see that the depth sensation can be perceived even from the two dimensional image that is displayed without using the effect of binocular parallax, when it was effectively displayed moving along the virtual plane in the dome environment. Therefore, in this study, the layered image method [8] that represents the three-dimensional space by using the twodimensional layered image was proposed. Figure 8 shows the concept of the layered image representation method [9].

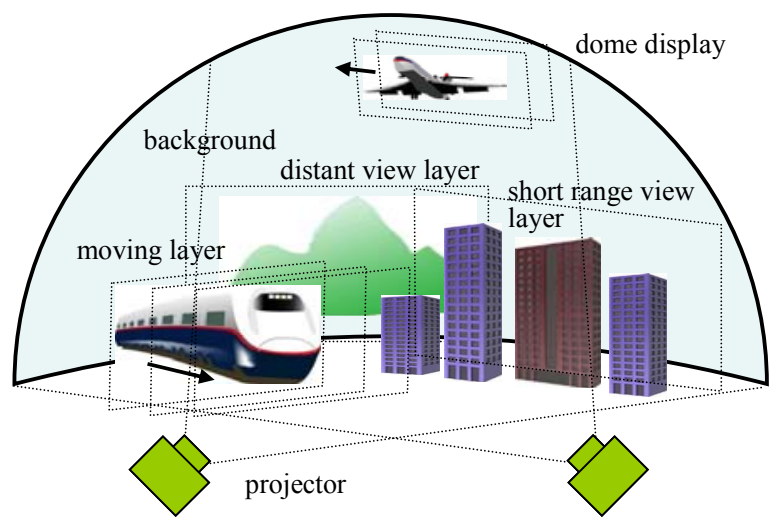

Figure 8. Concept of layer-based dome image. 
This method constructs the image space by placing the layered image elements such as the photograph, the video, or the rendered $\mathrm{CG}$ image in the three-dimensional space. In this method, it is expected that the difference of the distances from the layers can be perceived by the audience based on the principle described in previous chapter and the image space can be represented, by giving the movement of image elements or the movement of camera work.

In general, the cost of creating the dome contents was very high, because not only the image within the frame but also omni-directional image must be created. Particularly, this problem would be severe, when the dome contents were created using the three-dimensional computer graphics models. However, in this method, it is possible to create the contents with low-cost, because the image space is constructed by placing the layered images without making the three-dimensional models.

Figure 9 shows the example of the image space that was created using the layered image representation method. These figures show that the layer-based space can be perceived as the three-dimensional image from the correct view position of the audiences, though it consists of twodimensional layered images.

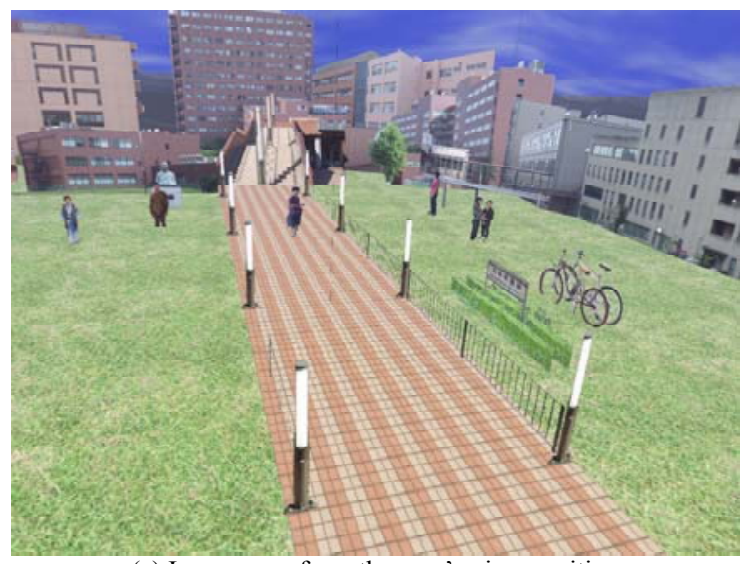

(a) Image seen from the user's view position

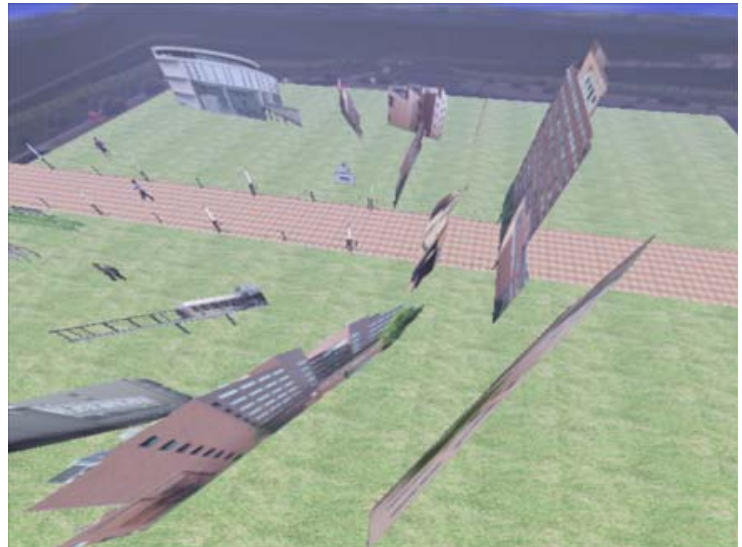

(b) Image seen from the sky

Figure 9. Example of layer-based image
In this study, several dome image contents were actually created using this method. Figure 10 shows the examples in which the contents of "Walk in University Campus" and "Guide of Tsukuba City" are being projected in the planetarium environment. These contents were created using the three-dimensional effects such as moving the objects or moving the view position dynamically. Then, the audience could feel the three-dimensional sensation from the image in the dome environment.

In these examples, it was possible to create the dome image contents in short development time. However, in the actual production of the layer-based image contents, the design of the image space in which the audiences can utilize their depth sensation effectively is a key issue. For example, some restriction is necessary for the area in which the image elements and the view position can be moved, so that the audience would not perceive the structure of the twodimensional layers in the image space.

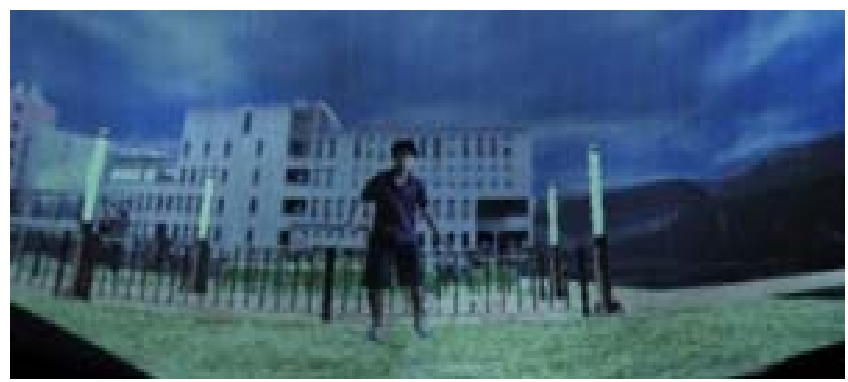

(a) "Walk in University Campus"

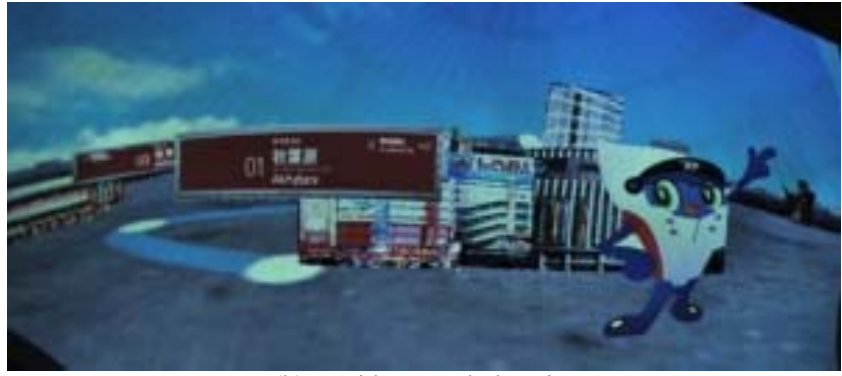

(b) "Guide to Tsukuba City"

Figure 10. Example of dome image contents projected in planetarium

\section{SCENARIO DESCRIPTION LANGUAGE}

Though the development cost of omni-directional image can be greatly reduced by using the layered image representation method, it is also necessary that the contents can be produced without the programming so that the usual contents creators can produce the dome image contents. Therefore, in this study, the scenario description language that defines the scenario of dome image contents and the rendering program that renders the image of contents that was described using the scenario language were developed.

Figure 11 shows the example of the description in the developed scenario description language. In order to produce the dome image contents, it is necessary to describe the scenario along the time axis as well as to design the omnidirectional space. This scenario language consists of two 
parts that are the definition of the image elements and the description of the scenario along time axis. In the part of the definition of the image elements, the file name, size, and the default position and attitude of the photo images and the movies are defined. In this case, it is possible to group some image elements, so that the same operation can be applied to the plural images in the group. In the part of the description of the scenario along the time axis, several scenes are described sequentially. In this case, visibility value, movement path, and movement time of the image elements as well as of the view position are described along the time axis in each scene.

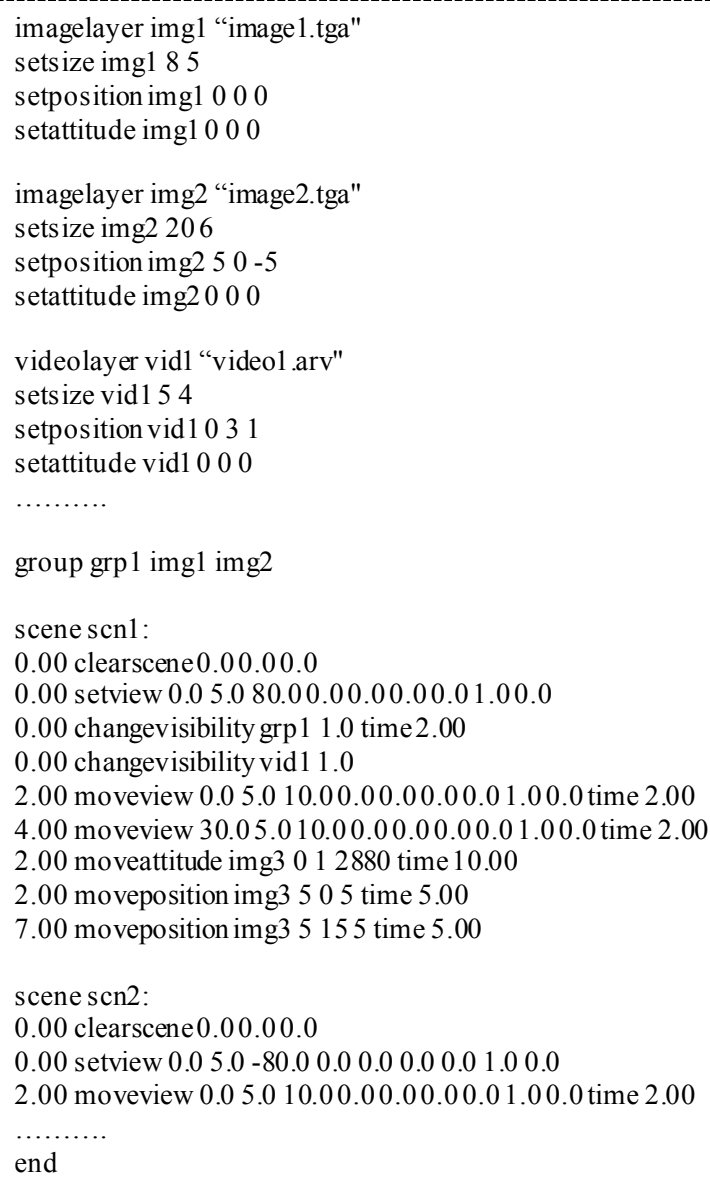

Figure 11. Example of scenario description language.

In order to display the contents that were described using the scenario language in the dome environment, the renderer program corresponding to this language is necessary. This renderer program consists of the parsing and the rendering functions that contain the distortion correction function for the contents described using the scenario description language. The distortion correction is performed by calculating the transformation parameter based on the camera calibration and applying it to the displayed image [10].
By using this method, the contents creator has become free from both the programming and image projection in the dome environment, and he can concentrate on only making scenario. However, in order to arrange the layered images and define the movement of them in the omni-directional space, it is necessary for the creator to consider the scenario in the three-dimensional coordinate system. In the future work, we are planning to develop an authoring tool that outputs the scenario description to facilitate the space design for the creators to solve this problem.

\section{CONCLUSIONS}

In this study, psychophysical experiment was conducted to evaluate the depth sensation that was felt in the dome environment quantitatively. From the result, it was shown that the perceived depth sensation can be controlled by changing the size of the geometrically corrected image and moving it with the background. Based on this result, the layered image representation method was proposed. And in addition, the scenario description language and the renderer program were developed so that the usual creator can produce the dome image contents easily without the programming. This paper discussed the basic phenomenon in which we can perceive the depth sensation from the image moving right and left in the dome environment. Future work will include systematizing the knowledge of effective representation method of the dome image by conducting psychophysical experiments in various conditions as well as developing the authoring tool to generate the scenario described using the scenario language easily.

\section{ACKNOWLEDGMENT}

This study was funded partly by Ministry of Internal Affairs and Communications (SCOPE, No.061303034) and supported by Keio University Global COE (Center of education and research of symbiotic, safe and secure system design) Program. And we would like to thank Prof. Yoshikazu Nakajima in the University of Tokyo, Prof. Takuro Kayahara in Miyagi University, Dr. Takeharu Senoo in Kyushu University and Masahiro Hayashi in Toyota Motor Corporation for their supports.

\section{REFERENCES}

[1] E. Lantz: The Digital Planetarium, Proc. of 2002 International Planetarium Society Conference, 2002

[2] K.C. Yu: Digital Full-Domes: The Future of Virtual Astronomy Education, The Planetarium, pp.6-11, 2005

[3] IEEE Canada. IMAX Technical Fact Sheet http://ieee.ca/millennium/imax/imax_technical.html

[4] Shibano N, Hareesh P.V, Hoshino H, Kawamura R, Yamamoto A, Kashiwagi $M$ and Sawada K: CyberDome: PC Clustered Hemi Spherical Immersive Projection Display, Proc. of 2003 International Conference on Artificial Reality and Telexistence (ICAT2003), pp.17, 2003.

[5] Katsuyuki Egawa: The Japan Pavilion Nagakute "Earth Vision" : The first images on a 360-degree, perfectly spherical screen in the world, IEICE technical report. Electronic information displays, Vol.105, No.392, pp.7-10, 2005 (in Japanese). 
[6] S. Otsuka, Depth from Motion Parallax in the Peripheral Visual Field, IEIC Technical Report, Vol.100, No.109, pp.37-39, 2000.K. Elissa, "Title of paper if known," unpublished.

[7] Takeharu Seno, Masahiro Hayashi, Tetsuro Ogi, Takao Sato: Virtual Depth Effects for Non-Stereoscopic Dome Images -The Estimation of the Depth Effects of the Dome Image by Psychophysics, 2008 ASIAGRAPH Proceedings, Vol.2, No.1, pp.121-126, Shanghai, 2008.
[8] J. Wang, E. Adelson: Layered Representation for Motion Analysis, Proc. of the IEEE Computer Society Conference on Computer Vision and Pattern Recognition 1993, pp. 361-366, 1993.

[9] Tetsuro Ogi, Masahiro Hayashi: Dome Image Contents based on Layered Image Representation, ASHIGRAPH 2007 in Tokyo Proceedings, Vol.1, No.2, pp.113-118, 2007.

[10] Zhengyou Zhang: A Flexible New Technique for Camera Calibration, IEEE Transactions on Pattern Analysis and Machine Intelligence, Vol.22, No.11, pp.1330-1334, 2000. 